\title{
Time/Frequency Analysis of Impulsive Noise on Powerline Channels
}

\author{
Gautier Avril', Mohamed Tlich², Fabienne Moulin', \\ Ahmed Zeddam $^{1}$, Fabienne Nouvel ${ }^{3}$ \\ 'Orange Labs - 2 Av. Pierre Marzin - 22307 Lannion, France \\ 2 Teamlog France \\ ${ }^{3}$ IETR - 20, av. des buttes de Coësmes, 35000 Rennes \\ gautier.avril $\omega$ orange-ftgroup.com
}

\begin{abstract}
Powerline communication systems are used to transmit audio and video transmissions with delay, datarate and QoS requirements. However, the Powerline medium is shared with other devices connected to any socket. When switching, and even during normal operation, these devices can generate some noises (stationary, cyclo-stationary \& impulsive). A good knowledge of these noises is essential to counter them and to ensure a fair quality of service. This study describes an new method to monitor impulsive noise, which tries to be very close to the mechanism of Powerline modems, so the impact of these noises is better evaluated
\end{abstract}

Keywords-Powerline, Impulsive noise, Time/Frequency Analysis, Spectrogram

\section{Introduction}

Powerline Systems are one of the main technologies to build a Home Network. These technologies are low cost, easy to use and offer the possibility to transmit high data rate with associated services (internet, high definition video, video on demand...). Some of these services, especially video and audio services, require a sufficient quality of service. However, the powerline medium used by powerline modem is shared with a lot a devices which may generate noises (stationary, cyclo-stationnary \& impulsive noise). Impulsive Noise (IN) is one of the main sources of interference which causes bit errors in power line communication (PLC) systems. Sources of these impulses are multiple: commutation of switches, power supplies... Some studies tried to classify their length, the bandwidth they use and interarrival time into different classes [ZIM00], [DEG03], [BAL03].

To counter these noises, Error Correction Codes (ECC) associated with interleaving and Automatic Repeat reQuest (ARQ) are generally used. Indeed, the power of these impulses makes it difficult to estimate impacted data. Different studies proved that impulsive noise varies from some $\mu$ s to some ms and that in-

Please use the following format when citing this chapter:

Avril, (i., Tlich, M., Moulin, F., 7eddam, A., Nouvel, F., 2007, in IFIP International Federation for Information Processing, Volume 256. Home Networking, Al Agha, K., Carcelle, X., Pujolle, G.. (Boston: Springer), pp. $143-150$. 
terarrival time can go up to a few seconds. So ECC length, interleaving and ARQ scheme are designed to neutralize these kinds of noises.

However, time, frequency and amplitude characteristics of impulsive noise can't be treated separately. Indeed, a long noise with very high amplitude may have a Power Spectral Density (PSD) concentrated on a small number of frequencies so the perturbation may be insignificant. On the contrary, a very short noise may affect a whole OFDM symbol... Moreover, PSD of the impulse may often vary between the beginning and the end of the impulse. To get a better estimation of the impact of impulsive noise, we bring together frequency and time, leading to a Time/Frequency analyzer for impulsive noise.

To have sufficient data for this analyzer, about 17 houses in France were monitored which provide us about 55000 noise acquisitions!

\section{- Experimental Hardware Configuration}

Measurements of impulsive noise are carried out, in time domain, by means of a numerical scope, as shows Figure 1.

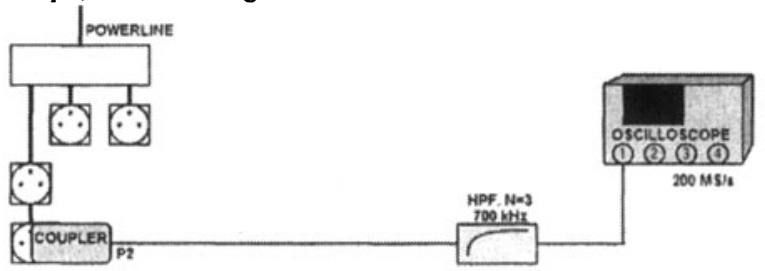

Fig. 1. Impulsive noise measurement hardware.

On each site, the acquisition of the impulsive noises is carried over 24 to 48 hours duration. An impulsive noise is recorded over a $48 \mathrm{~ms}$ duration when its amplitude exceeds the trigger level. This level depends on the stationary noise present on the line and can thus vary from one site to another. For the majority of sites, the trigger level is fixed to $50 \mathrm{mV}$.

The noise is recorded on several scope channels with different sensitivities. This makes it possible to have at the same time a good sensitivity for the lowamplitude noises and not to clip the strong-amplitude noises.

Let's note, finally, that the sampling rate was fixed to $250 \mathrm{MHz}$.

\section{General Observation}

The observation of the measured impulsive noises highlighted two principal noise categories:

- Asynchronous impulsive noises, for which the duration varies from few microseconds to few milliseconds and whose frequency of appearance is random.

- Pseudo-stationary periodic impulsive noises synchronous with the sector frequency. Their repetition rate is thus of $50 \mathrm{~Hz}$ or $100 \mathrm{~Hz}$. 
These two categories are part of the five noise categories (stationary noise included/understood) established by Zimmermann and Dostert in [ZIM00]. Impulsive and pseudo stationnary noise can be seen on Figure 1 .

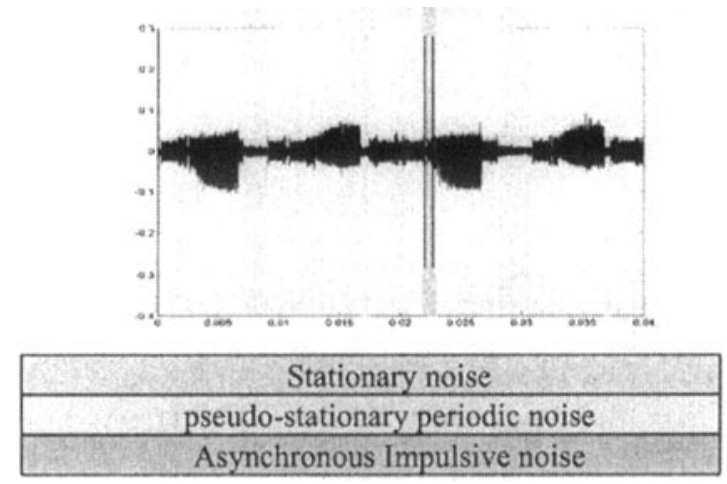

Fig. 2. Temporal observation of PLC noise.

In order to illustrate the importance of characterizing the impulsive noises by a system approach, we consider the example of an asynchronous impulsive noise generated by a computer screen. This noise, shown in Figure 3 in time domain and in Figure 4 in frequency one, lasts almost $50 \mathrm{~ms}$ and its energy is mainly concentrated in low frequencies.

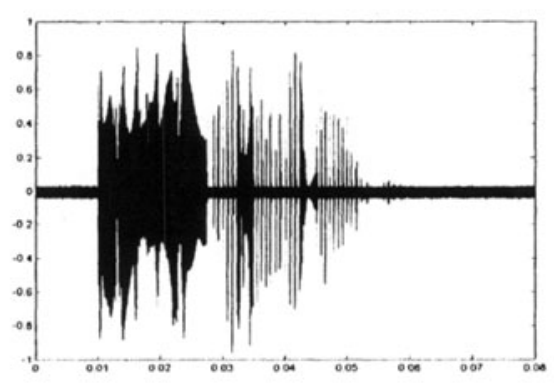

Fig. 3. Impulsive noise generated by a computer screen (time domain).

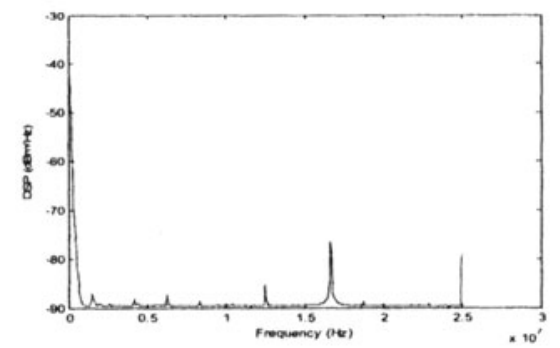

Fig. 4. Impulsive noise generated by a computer screen (frequency domain). 
As Figure 5 shows, when this noise is treated according to the system approach, i.e. cut out in PLC OFDM symbols for which the FFT is applied, we observe that the noise PSD differs from its PSD calculated over its total duration, and also varies from an OFDM symbol to another.

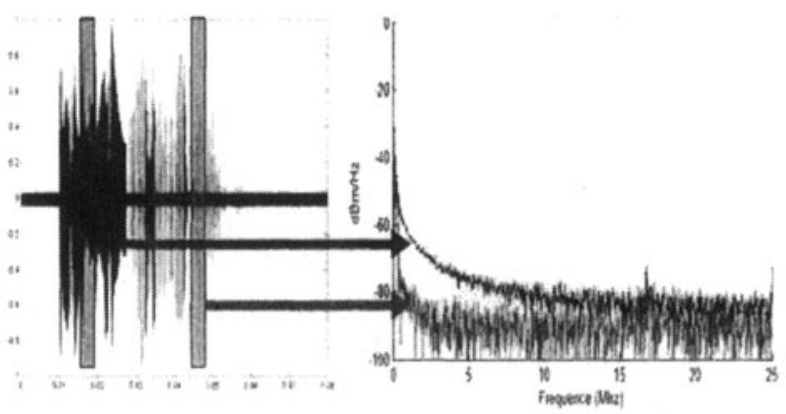

Fig. 5. PSD of an impulse at 2 different points

\section{- Spectrogram Representation}

This observation brought us to think about a spectrogram representation of the impulsive noises. This representation makes it possible to observe the noise in time and in frequency as it is really perceived by a communication system.

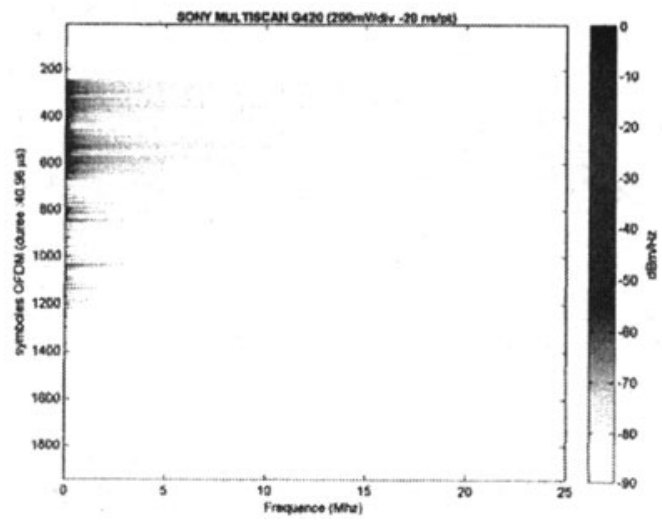

Fig. 6. Time/frequency spectrogram of the impulse

Figure 6 gives the time/frequency spectrogram of the same impulsive noise generated by a computer screen. For each OFDM symbol whose duration is equal to $40.96 \mu \mathrm{s}$, its PSD is calculated and its noise strength is represented by suitable colour darkness. 
This representation has the advantage of determining:

- The number of symbols really affected by the impulsive noise.

- The OFDM carriers affected by the impulsive noise.

Such kind of representation makes it possible to identify the signatures of various disturbers.

In particular, this analysis was also performed on pseudo-stationary periodic noise synchronous with the $50 \mathrm{~Hz}$ frequency, which strongly affects powerline systems ([CAI05] [KAT06]).

In Figure 7 is reported the impulsive noise spectrogram generated by a low consumption lamp, while Figure 8 shows the PSD of this noise calculated on two time-distant OFDM symbols. We can easily distinguish a $4 \mathrm{~ms}$ duration pulse, which repeats every $10 \mathrm{~ms}$.

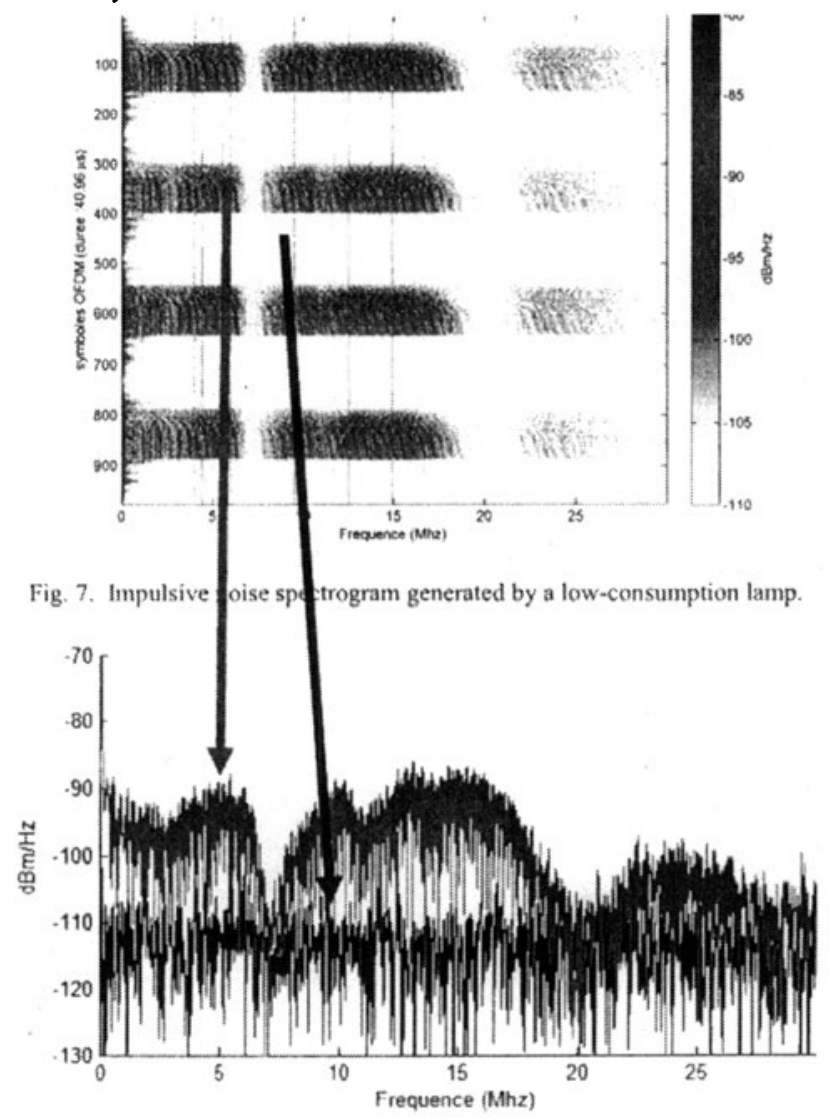

Fig. 8. PSD of the Fig. 7 noise on two time-distant OFDM symbols. 


\section{- Impact on Powerline Systems}

It is very difficult to determine the impact of asynchronous impulsive noise on powerline systems. Indeed, Automatic Repeat reQuest (ARQ) is used on these systems and is very efficient to fight most of powerline noises.

To limit the effect of Automatic Repeat reQuest, we send a fixed $12 \mathrm{Mb} / \mathrm{s}$ HDTV UDP stream on a given powerline link which allows up to $13-14 \mathrm{Mb} / \mathrm{s}$ datarate. This approach is useful because HomePlug AV (HPAV) modems use ARQ even for UDP stream.

With only a 1 or $2 \mathrm{Mb} / \mathrm{s}$ datarate margin, it becomes more difficult for HomePlug modems to reemit impacted data.

Some of previously recorded noises were injected with an Arbitrary Waveform Generator (AWG) as shown on Fig. 9.

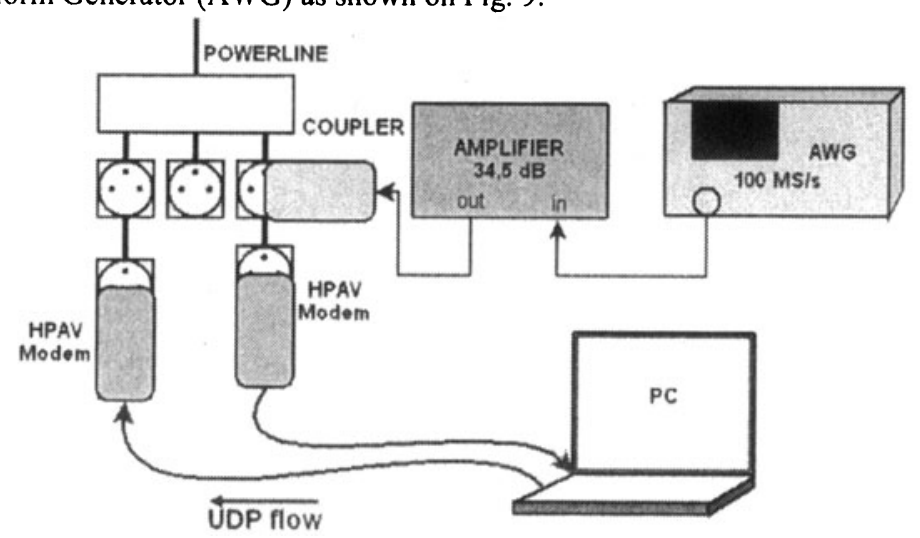

Fig. 9. Injection of recorded noises.

Table I presents the impact of some noises on the UDP transfer.

TABLE I

MEASURED PERIODIC NOISES IN 5 SITES

\begin{tabular}{cccc}
\hline $\begin{array}{c}\text { Noise Re- } \\
\text { cording }\end{array}$ & $\begin{array}{c}\text { Impulse } \\
\text { length }(\mu \mathrm{s})\end{array}$ & $\begin{array}{c}\text { Affected } \\
\text { points }\end{array}$ & $\begin{array}{c}\text { UDP Packet } \\
\text { loss }\end{array}$ \\
\hline 1 & $<41$ & 125 & 0 \\
2 & $<41$ & 2068 & 0 \\
3 & 82 & 1568 & 0 \\
4 & 4915 & 765 & 1920 \\
5 & 28795 & 79802 & 19862 \\
6 & 36740 & $7,7 \mathrm{e}^{7}$ & 22056 \\
7 & 37068 & $4,1 \mathrm{e}^{6}$ & 25432
\end{tabular}


We see that the impact on UDP transfer is more linked with the length of the impulse than with the number of affected points. However, this only proves that ARQ is dependant of the length of the impulse. Our approach may however be used to evaluate error correction codes algorithms.

The impact of pseudo stationary impulsive and pseudo-stationary periodic noise synchronous with the $50 \mathrm{~Hz}$ was also studied.

For every device which produces impulsive noise, we make statistics on each point (one frequency for one OFDM symbol) affected by impulsive noise per $50 \mathrm{~Hz}$ cycle. These results are put together with the datarate obtained with a Homeplug $85 \mathrm{Mbits} / \mathrm{s}$ modem

Table II presents an example of some of these results.

TABLE II

MEASURED PERIODIC NOISES IN 5 SITES

\begin{tabular}{ccccc}
\hline \hline Device & $\begin{array}{c}\text { impulse length } \\
\text { per 20 ms }(\mu \mathrm{s})\end{array}$ & $\begin{array}{c}\text { Amplitude } \\
(\mathrm{V})\end{array}$ & $\begin{array}{c}\text { Affected } \\
\text { points }\end{array}$ & $\begin{array}{c}\text { HomePlug } \\
\text { Datarate loss }\end{array}$ \\
\hline 1 & 120 & 2,3 & 682 & $-9 \%$ \\
2 & 1000 & 6,0 & 3286 & $-11 \%$ \\
3 & 1000 & 3,3 & 11535 & $-24 \%$ \\
4 & 1500 & 1,1 & 14488 & $-23 \%$ \\
5 & 120 & 0,5 & 58 & - \\
6 & 1000 & 0,9 & 568 & $-7 \%$ \\
7 & 4000 & 4,1 & 45454 & $-73 \%$ \\
8 & 7000 & 1,3 & 42023 & $-56 \%$ \\
9 & 1200 & 3,3 & 17045 & $-28 \%$ \\
10 & 9000 & 5,1 & 32010 & $-72 \%$ \\
11 & 160 & 1,7 & 470 & $-8 \%$
\end{tabular}

This table shows that more than impulse length, amplitude or PSD, the knowledge of the number of affected points may be used to predict the impact of noise on a PLC modem.

By looking at the worst recorded impulse, we can imagine new techniques to improve the next generation of Powerline modems. 


\section{- Conclusion}

This system-based analysis of the impulsive noise is very useful to evaluate the impact of impulses on powerline modem as it processes the data as the modems do. Here, the impulses are divided into segments whose durations are equal to the Homeplug AV symbol duration. The spectrogram representation, selected for this approach, makes it possible to visualize the impact which the noise would have on the transmission (visualization of the symbols and OFDM carriers potentially affected by the impulsive noise).

Moreover, general statistics are obtained and allow us to propose new algorithms to better fight impulsive noise for the next generation of PLC.

\section{- References}

[HPAV] HomePlugAV Specification.

[ZIM00] An Analysis of the Broadband Noise Scenario in Power-Line Networks, L. Zimmermann, K. Dostert, ISPLC 2000.

[BAL03] Potential Limits on Power-Line Communication over Impulsive Noise Channels V.B. Balakirsky, A.J.H. Vinck, ISPLC 2003.

[CAI05] Fundamentals of the Cyclic Short-Time Variation of Indoor Powerline Channels, F.J Caiiete, L. Diez, J.A. Cortes, J.T. Entrambasaguas, J.L. Carmona, ISPLC 2005.

[KAT06] Channel Adaptation based on Cyclo-Stationary Noise Characteristics in PLC Systems. S. Katar, B. Mashburn, K. Afkhamie, H. Latchman, R. Newman. ISPLC 2006.

[DEG03] Impulsive Noise on Indoor Power lines : Characterization and Mitigation of its Effect on PLC Systems V. Degardin, M. Lienard, P. Degauque, A. Zeddam, F. Gauthier, EMC 2003. 